УДК 512.542

\title{
On Intersection of Primary Subgroups in the Group $\operatorname{Aut}\left(\mathbf{F}_{4}(2)\right)$
}

\author{
Viktor I. Zenkov* \\ Institute of Mathematics and Mechanics UB RAS \\ Kovalevskoi, 16, Ekaterinburg, 620990 \\ Ural Federal University \\ Mira, 19, Ekaterinburg, 620990 \\ Russia \\ Yakov N. Nuzhin ${ }^{\dagger}$ \\ Institute of Mathematics and Computer Science \\ Siberian Federal University \\ Svobodny, 79, Krasnoyarsk, 660041
}

Russia

Received 20.05.2017, received in revised form 29.12.2017, accepted 20.01.2018

It is proved that, in a finite group $G$ which is isomorphic to the group of automorphisms of the Chevalley group $F_{4}(2)$, there are only three possibilities for ordered pairs of primary subgroups $A$ and $B$ with condition: $A \cap B^{g} \neq 1$ for any $g \in G$. We describe all ordered pairs $(A, B)$ of such subgroups up to conjugacy in the group $G$ and in particular, we prove that $A$ and $B$ are 2-groups.

Keywords: finite group, almost simple group, primary subgroup.

DOI: 10.17516/1997-1397-2018-11-2-171-177.

\section{Introduction and preliminaries}

Let $G$ be a finite group and $A$ and $B$ be its subgroups. By definition, $M$ is the set of subgroups that are minimal by inclusion among all subgroups of type $A \cap B^{g}, g \in G$, and $m$ consists of those elements of the set $M$ whose order is minimal. Denote by $\operatorname{Min}_{G}(A, B)$ (resp. $\left.\min _{G}(A, B)\right)$ the subgroup, generated by the set $M$ (resp. $m$ ). First this kind of groups was introduced in [1]. Evidently, $\min _{G}(A, B) \leqslant \operatorname{Min}_{G}(A, B)$ and the following three conditions are equivalent: a) $A \cap B^{g} \neq 1$ for any $g \in G$; b) $\operatorname{Min}_{G}(A, B) \neq 1$; c) $\min _{G}(A, B) \neq 1$.

If $S \in \operatorname{Syl}_{p}(G)$ then subgroups $\min _{G}(S, S) \neq 1$ can be described in many interesting cases. It give us a description of pairs of subgroups $(A, B)$ with the condition $\min _{G}(A, B) \neq 1$ for primary subgroups and sometimes for nilpotent subgroups $A$ and $B$. For example, in [2, Theorem 1] it is proved that $\operatorname{Min}_{G}(A, B) \leqslant F(G)$ for any pair of abelian subgroups $A$ and $B$ of $G$, where $F(G)$ is the Fitting subgroup of $G$ (the greatest normal nilpotent subgroup of $G$ ).

It was proved in [3] that if $G$ is an almost simple group with socle $L_{2}(q), q>3$, and $S \in \operatorname{Syl}_{p}(G)$, then $\min _{G}(S, S)=\operatorname{Min}_{G}(S, S)=S$ for the Mersenne prime $q=2^{n}-1$, and the equalities $\min _{G}(S, S)=\operatorname{Min}_{G}(S, S)=1$ hold for all others $q$, exception $q=9$. For $q=9$

\footnotetext{
*v1i9z52@mail.ru

$\dagger$ nuzhin2008@rambler.ru

(C) Siberian Federal University. All rights reserved
} 
the subgroup $\min _{G}(S, S)$ is isomorphic to the dihedral group $D_{16}$ and it has index 2 in the group $S$. The exceptional case is important for our paper, therefore we mention corresponding result of [4].

Let socle of $G$ be isomorphic to $L_{2}(9) \simeq A_{6}$ and $S \in S y l_{p}(G)$. Then $\min _{G}(S, S)=1$ for $p>2$, but for $p=2$ the equality $\min _{G}(S, S)=1$ holds for all $G$, exception $G=\operatorname{Aut}\left(A_{6}\right)$. In exception case $\min _{G}(S, S)=\langle i, j\rangle \simeq D_{16}, i^{2}=j^{2}=1$, and $\left|C_{S}(i)\right|=\left|C_{S}(j)\right|=8$, where $i, j$ belongs to $S \backslash S \cap S o c(G)$ and $j$ (resp. $i$ ) induces field (resp. diagonal) automorphism of the $\operatorname{group} \operatorname{Soc}(G)$.

We need some information about subgroups of the Chevalley group $F_{4}(2)$. Let $r_{2}$ and $r_{3}$ be fundamental roots of the root system of type $F_{4}$ which generate subsystem of type $B_{2}$. Denote by $P_{\{2,3\}}$ the parabolic subgroup which is generated by monomial elements $n_{r_{2}}, n_{r_{3}}$ and unipotent subgroup $U$ corresponding positive roots. The subgroup $P_{\{2,3\}}$ is invariant under graph automorphism $\tau$ of order 2, its Levy subgroups $L$ is isomorphic to the Chevalley group $B_{2}(2)$. The product $L\langle\tau\rangle$ is isomorphic to the group $A u t\left(A_{6}\right)$. We prove the following theorems.

Theorem 1. Let $G$ be a finite group with socle $F_{4}(2), S$ be a Sylow 2-subgroup of $G$ and $\operatorname{Min}_{G}(S, S) \neq 1$. Then $G \simeq \operatorname{Aut}\left(F_{4}(2)\right)$ and

$$
\min _{G}(S, S)=O_{2}\left(P_{\{2,3\}}\right) \cdot \min _{L\langle\tau\rangle}\left(S_{1}, S_{1}\right)
$$

where $S_{1}$ is a Sylow 2-subgroup of the group $L\langle\tau\rangle$ and $\min _{L\langle\tau\rangle}\left(S_{1}, S_{1}\right) \simeq D_{16}$.

Theorem 2. Let $A, B$ be p-subgroups of a finite group $G$ with socle $F_{4}(2)$ and $S$ be a Sylow 2-subgroup of $G$. Then the following are equivalent:

1) $\operatorname{Min}_{G}(A, B) \neq 1$;

2) $p=2, G \simeq \operatorname{Aut}\left(F_{4}(2)\right)$ and up to conjugacy in the group $G$ the ordered pair $(A, B)$ lies in the set $\left\{(S, S),\left(\min _{G}(S, S), S\right),\left(S, \min _{G}(S, S)\right)\right\}$.

\section{Notations and preliminary results}

Further, $G$ be a finite group, $A$ and $B$ be its subgroups. The sets $M, m$ and the subgroups $\operatorname{Min}_{G}(A, B), \min _{G}(A, B)$ as in the introduction. Others notations are standard for group theory. For example, $\operatorname{Syl}_{p}(G)$ is the set of all Sylow p-subgroups of the group $G$, and $\operatorname{Soc}(G)$ is the socle of $G$ (the minimal normal subgroup of the group $G$ ).

Lemma 2.1 ( [4]). Let $\operatorname{Soc}(G)=A_{6}, S \in \operatorname{Syl}_{p}(G)$ and $\min _{G}(S, S) \neq 1$. Then $G=A u t\left(A_{6}\right)$, $p=2$ and $\min _{G}(S, S)=\langle i, j\rangle \simeq D_{16}$, where $i^{2}=j^{2}=1,\left|C_{S}(i)\right|=\left|C_{S}(j)\right|=8$, the order of each elements of $m$ is equal to 2 and the subgroup $\langle i, j\rangle$ cowers quotient groups $G / G^{\prime}$.

Lemma 2.2 ([4]). Let $A, B$ be p-subgroups of $G, \operatorname{Soc}(G)=A_{6}, S \in S y l_{p}(G)$ and $\min _{G}(A, B) \neq 1$. Then $G=\operatorname{Aut}\left(A_{6}\right), p=2$ and $(A, B) \in\left\{(S, S),\left(\min _{G}(S, S), S\right),\left(S, \min _{G}(S, S)\right)\right\}$.

Lemma 2.3 ( [1]). Let $G \geqslant G_{1} \unrhd G_{2}, G_{1} \geqslant A, G \geqslant B$. Suppose that $G_{2} \cap B^{h}=1$ for some $h \in G$ and in the quotient group $\overline{G_{1}}=G_{1} / G_{2}$ we have $\bar{A} \cap\left(\overline{G_{1} \cap B^{h}}\right)^{\bar{f}}=\overline{1}$ for some $\bar{f} \in \overline{G_{1}}$. Then $A \cap B^{g}=1$ for some $g \in G$.

In conclusion of this part we note a simple example the group $G$ with subgroups $A$ and $B$ for which $\operatorname{Min}_{G}(A, B) \neq \min _{G}(A, B)$.

Let $G$ be the symmetric group on the four symbols and $S \in S y l_{2}(G)$. Then $S \simeq D_{8}$ and $O_{2}(G) \simeq Z_{2} \times Z_{2}$. Take the subgroup $S$ as $A$ and as $B$ we take the subgroup of order four of $S$, which not belongs to $O_{2}(G)$. Then $\left|B^{g} \cap O_{2}(G)\right|=2$ for any $g \in G$. Since $A \cap B=B$ and $\left|A \cap B^{f}\right|=2$ for $B^{f} \not A$, then $M=\left\{B, B^{f} \cap O_{2}(G), B^{f^{2}} \cap O_{2}(G)\right\}$, where $|f|=3$. Therefore $\operatorname{Min}_{G}(A, B)=S \neq O_{2}(G)=\min _{G}(A, B)$. 


\section{Some properties of the group $\operatorname{Aut}\left(F_{4}(q)\right)$}

Further, $\Phi$ is a reduced indecomposable root system, $\Pi=\left\{r_{1}, \ldots, r_{l}\right\}$ is its set of fundamental roots, $\Phi^{+}$is the positive root system respect to $\Pi$, and also $\Phi^{-}=-\Phi^{+}$.

Denote by $\Phi(q)$ an adjoint Chevalley group of type $\Phi$ of rank $l$ over the finite field $\mathbb{F}_{q}$ of the order $q=p^{n}$, where $p$ is a prime. The group $\Phi(q)$ is generated by the root subgroups $X_{r}=\left\langle x_{r}(t) \mid t \in \mathbb{F}_{q}\right\rangle, r \in \Phi$, where $x_{r}(t)$ is the corresponding root element in the group $\Phi(q)$. We will need the following natural subgroups of the group $\Phi(q)$ : the unipotent subgroups $U=\left\langle X_{r} \mid r \in \Phi^{+}\right\rangle, V=\left\langle X_{r} \mid r \in \Phi^{-}\right\rangle$, the monomial subgroup $N=\left\langle n_{r}(t) \mid r \in \Phi, t \in \mathbb{F}_{q}^{*}\right\rangle$, the diagonal subgroup $H=\left\langle h_{r}(t) \mid r \in \Phi, t \in \mathbb{F}_{q}^{*}\right\rangle$ and the Borel subgroup $B=U H$. Here, $\mathbb{F}_{q}^{*}$ is the multiplicative subgroup of the field $\mathbb{F}_{q}$ and $n_{r}(t)=x_{r}(t) x_{-r}\left(-t^{-1}\right) x_{r}(t), h_{r}(t)=n_{r}(t) n_{r}(-1)$. We set also $I=\{1,2, \ldots, l\}$.

Overgroups of the Borel subgroup $B$ and conjugate with them are called parabolic. Due to familiar result of J.Tits, parabolic subgroups containing subgroup $B$ are $P_{J}=\left\langle B, n_{r_{j}}(1) \mid j \in J\right\rangle$, where $J \subseteq I$.

Lemma 3.1 ( [5], Lemma 5). Fix a monomial element $n_{0}$ with condition $U^{n_{0}}=V$ and a positive integer $i \in I$. Set $n=n_{0} n_{r_{i}}(1)$. Then $U \cap U^{n}=X_{r_{i}}$.

For $l=1$ the root subgroup $X_{r_{i}}$ coincides with a Sylow $p$-subgroup of the group $\Phi(q)$ and in this case in the Lemma 3.1 the element $n$ is diagonal.

Further, $\Pi=\left\{r_{1}, r_{2}, r_{3}, r_{4}\right\}$ is a fundamental root system of type $F_{4}$, moreover $r_{1}, r_{2}$ are short roots and $r_{2}+r_{3}$ is a root. The graph automorphism $\tau$ of the Chevalley groups $F_{4}(2)$ is defined correctly by symmetry of order 2 of the Coxeter graph of type $F_{4}$, which induces the bijection $r \rightarrow \bar{r}$ of the root system of type $F_{4}$ to itself such that $\overline{-r}=-\bar{r}$ [6, Lemma 12.3.2]. Note, by the way, that root system of type $F_{4}$ is the union subsystems $\Phi_{1}$ and $\Phi_{2}$ of type $B_{4}$ and $C_{4}$ respectively and $\overline{\Phi_{1}}=\Phi_{2}$ (see, for Example, [7]).

Lemma 3.2. Let $U, V$ be the unipotent subgroups of the group $F_{4}(2)$ with the graph automorphism $\tau$ of order 2 as above. Then $S=U \lambda\langle\tau\rangle$ is a Sylow 2-subgroup of the group $\operatorname{Aut}\left(F_{4}(2)\right)=F_{4}(2)\langle\tau\rangle$ and there is an unique monomial element $n_{0}$ such that $S \cap S^{n_{0}}=\langle\tau\rangle$.

Proof. Just the last equality requires justification. In the group $F_{4}(2)$ there is an unique monomial element $n_{0}$ such that $U^{n_{0}}=V$. Since $X_{r}^{n_{0}}=X_{-r}, X_{r}^{\tau}=X_{\bar{r}}$ and $\overline{-r}=-\bar{r}$, then $n_{0} \tau=\tau n_{0}$. Hence, $S \cap S^{n_{0}}=\langle\tau\rangle$.

Lemma 3.3. Let $S$ be as in Lemma 3.2. Then in the group $F_{4}(2)$ there is a monomial element $n$ such that $S \cap S^{n}=X_{r_{1}}$.

Proof. Let a monomial element $n_{0}$ be as in Lemma 3.1 and $n=n_{0} n_{r_{1}}(1)$. By Lemma 3.1 we have $U \cap U^{n}=X_{r_{1}}$. Now using the equalities $n_{0} \tau=\tau n_{0}$ (see proof of Lemma 3.2) and $n_{r_{1}}(1) \tau n_{r_{1}}(1)=n_{r_{1}}(1) n_{r_{4}}(1) \tau$ we obtain the assertion of lemma $S \cap S^{n}=X_{r_{1}}$.

Lemma 3.4. Let $i=1$ or 4 and $P=P_{I \backslash\{i\}}$ be a maximal parabolic subgroup of the group $F_{4}(q)$. Then $\left\langle X_{r_{i}}^{U}\right\rangle=O_{p}(P)$.

Proof. Let $P=P_{I \backslash\{4\}}$. Then we have equality

$$
O_{p}(P)=\left\langle X_{r} \mid r=c_{k} r_{k}+\cdots+c_{4} r_{4}, 1 \leqslant k \leqslant 4, c_{j} \geqslant 1\right\rangle .
$$

Further, for a root $r=a r_{1}+b r_{2}+c r_{3}+d r_{4}$ we will use the notation $a b c d$. Using this compact representation of roots and the table VIII for the root system of type $F_{4}$ in [8], we have equality

$$
O_{p}(P)=\left\langle X_{r} \mid r \in \Psi\right\rangle
$$


where

$$
\Psi=\{0001,0011,0111,1111,0211,0221,1211,1221,2211,1321,2221,2321,2421,2431,2432\} .
$$

Evidently, $X_{0001} \subseteq M$. For any $t, u \in \mathbb{F}_{q}$ the commutator formula of Chevalley gives the equalities

$$
\begin{aligned}
{\left[x_{0001}(t), x_{0010}(u)\right] } & =x_{0011}( \pm t u), & & {\left[x_{2211}(t), x_{0010}(u)\right]=x_{2221}( \pm t u), } \\
{\left[x_{0001}(t), x_{0210}(u)\right] } & =x_{0211}( \pm t u), & & {\left[x_{0221}(t), x_{1000}(u)\right]=x_{1221}( \pm t u) x_{2221}\left( \pm t u^{2}\right), } \\
{\left[x_{0211}(t), x_{0010}(u)\right] } & =x_{0221}( \pm t u), & & {\left[x_{1211}(t), x_{0010}(u)\right]=x_{1221}( \pm t u) x_{2432}\left( \pm t^{2} u\right), } \\
{\left[x_{0011}(t), x_{0100}(u)\right] } & =x_{0111}( \pm t u) x_{0211}\left( \pm t u^{2}\right), & & {\left[x_{1221}(t), x_{0100}(u)\right]=x_{1321}( \pm t u), } \\
{\left[x_{0111}(t), x_{1000}(u)\right] } & =x_{1111}( \pm t u), & & {\left[x_{1321}(t), x_{1000}(u)\right]=x_{2321}( \pm t u), } \\
{\left[x_{1111}(t), x_{0100}(u)\right] } & =x_{1211}( \pm t u), & & {\left[x_{2211}(t), x_{0210}(u)\right]=x_{2421}( \pm t u), } \\
{\left[x_{0211}(t), x_{1000}(u)\right] } & =x_{1211}( \pm t u) x_{2211}\left( \pm t u^{2}\right), & & {\left[x_{2221}(t), x_{0210}(u)\right] }
\end{aligned}
$$

Using these equalities, we successively obtain the inclusions $X_{r} \subseteq O_{p}(P)$ for all $r \in \Psi$. The conclusion of the lemma is also true for $i=1$ by the equality $P_{I \backslash\{4\}}^{\tau}=P_{I \backslash\{1\}}$.

\section{Some properties of Sylow p-subgroups of the groups of Lie type over fields of characteristic $p$}

Analogues of the subgroups $X_{r}, U, V, N, H, B, P_{J}$ of the Chevalley group $\Phi(q)$ in Section 3 are also defined for twisted Chevalley group ${ }^{n} \Phi(q)$. In this section, $G(q)$ is a group of Lie type over a finite field of order $q$ of characteristic $p$, where $G=\Phi$ or ${ }^{n} \Phi$. It is well known that any parabolic subgroup $P_{J}$ of the group $G(q)$ is a semidirect product with kernel $O_{p}\left(P_{J}\right)$ and a noninvariant factor $L$. A subgroup $L$ is called a Levi factor and it is isomorphic to the central product of groups of Lie type of smaller ranks over the initial field.

We will need the following strengthening of Lemma 3.13 from [3].

Lemma 4.1. The number of orbits under the action of conjugation by elements of $U$ on the set of subgroups $U^{g}$ of $G(q)$ with the condition $U \cap U^{g}=1, g \in G(q)$, is equal to one. Moreover, the length of this single orbit is $|U|$ and it consists of subgroups of the form $V^{u}, u \in U$.

Proof. Any element $g \in G(q)$ can be uniquely represented in the form $g=u n_{w} v$, where $u, v \in U, n_{w} \in N$, and $n_{w} v n_{w}^{-1} \in V$. Let $U \cap U^{g}=1$. Then $U \cap U^{n_{w}}=1$. Since $X_{r}^{n_{w}}=X_{w(r)}$, then $w\left(\Phi^{+}\right)=-\Phi^{+}$. Thus, any subgroup $U^{g}$ with the condition $U \cap U^{g}=1$ has the form $V^{u}$ for some $u \in U$. Since $N_{G(q)}(V)=H V$, then the number of subgroups of the form $V^{u}, u \in U$, with the condition $U \cap V^{u}=1$ is equal to $|U|$.

Lemma 4.2. $V \cap O_{p}\left(P_{J}\right)=1$ and the subgroup $V$ covers the Sylow p-subgroup in the quotient group $\overline{P_{J}}=P_{J} / O_{p}\left(P_{J}\right)$.

Proof. Since $O_{p}\left(P_{J}\right) \subseteq U$, and $U \cap V=1$, then $O_{p}\left(P_{J}\right) \cap V=1$. By virtue of the Levi decomposition $\left|S y l_{p}\left(\overline{P_{J}}\right)\right|=\left|P_{J} \cap V\right|$. Consequently, the subgroup $V$ covers the Sylow $p$-subgroup of the quotient group $\overline{P_{J}}=P_{J} / O_{p}\left(P_{J}\right)$.

\section{The proof of the Theorem 1}

Further in the proof, we use the notations of the Section 3 for subgroups and elements of the group $\operatorname{Aut}\left(F_{4}(2)\right)$. 
So, by the hypothesis of the theorem, $G$ is a finite group, $\operatorname{Soc}(G) \simeq F_{4}(2), S \in \operatorname{Syl}_{2}(G)$ and $\operatorname{Min}_{G}(S, S) \neq 1$. Since $\operatorname{Aut}\left(F_{4}(2)\right)=F_{4}(2)\langle\tau\rangle$ then it is possible only two cases: 1$) G=F_{4}(2)$; 2) $G=F_{4}(2)\langle\tau\rangle$.

The first case is not possible, because the Sylow 2-subgroups $U$ and $V$ of the group $G=F_{4}(2)$ have the unite intersection.

Let $G=F_{4}(2)\langle\tau\rangle$. Without loss of generality, we can assume that $S=U\langle\tau\rangle$. Let $g \in G$. Then $g=u_{1} n u_{2} \theta$, where $u_{1}, u_{2} \in U, n \in N$ and $\theta \in\langle\tau\rangle$. If $n \neq n_{0}$ then $U \cap U^{g} \neq 1$ and, consequently, $S \cap S^{g} \neq 1$. If $n=n_{0}$, then we have $S \cap S^{g}=\langle\tau\rangle$ by Lemma 3.2. Thus $S \cap S^{g} \neq 1$ for each $g \in G$ and moreover any element (subgroup) of the set $m$ for $A=B=S$ has order 2 . Set

$$
P=P_{\{2,3\}} .
$$

By Lemma 3.1 there is a monomial element $n \in F_{4}(2)$ such that $S \cap S^{n}=X_{r_{1}}$. By Lemma 3.4

$$
\left\langle X_{r_{i}}^{U}\right\rangle=O_{2}\left(P_{I \backslash\{i\}}\right)
$$

for $i=1$ or 4 . Since $\left\langle O_{2}\left(P_{I \backslash\{i\}}\right), O_{2}\left(P_{I \backslash\{i\}}^{\tau}\right)\right\rangle=O_{2}(P)$, then $O_{2}(P) \leqslant \min _{G}(S, S)$.

Let

$$
N=N_{G}(P)=P\langle\tau\rangle
$$

Then $O_{2}(N)=O_{2}(P)$ and

$$
\bar{N}=N / O_{2}(N) \simeq \operatorname{Aut}\left(A_{6}\right) .
$$

We choose an element $x \in G$ such that the intersection of cardinality 2

$$
D=S \cap S^{x} \in m
$$

does not lie in $O_{2}(N)$. (Such an element certainly exists, for example, as $x$, we can take the element $n_{0}$ from Lemma 3.1.) Since $O_{2}(N) \subseteq S$, then $O_{2}(N) \cap S^{x}=1$. Set

$$
S_{1}=N \cap S^{x} .
$$

By Lemma 4.3 , the subgroup $U^{x}$, and therefore by definition, the subgroup

$$
U_{1}=P \cap U^{x} \leqslant S_{1}
$$

covers a Sylow 2-subgroup of the factor group

$$
\bar{P}=P / O_{2}(P) \simeq S p_{4}(2) .
$$

Socle of the group $S p_{4}(2)$ is isomorphic to $A_{6}$, but $S p_{4}(2) \not A \operatorname{Aut}\left(A_{6}\right)$. Therefor $\min _{\bar{P}}\left(\bar{U}, \overline{U_{1}}\right)=\overline{1}$ by Lemma 2.1 . Hence, also $\min _{\bar{N}}\left(\bar{U}, \overline{U_{1}}\right)=\overline{1}$, since $|\bar{N}: \bar{P}|=2$ and $\bar{U}, \overline{U_{1}}<\bar{P}$.

We show that $S_{1} \neq U_{1}$. Suppose the contrary, let $S_{1}=U_{1}$. Then in the quotient group $\bar{N}$ we have

$$
\min _{\bar{N}}\left(\bar{S}, \bar{S}_{1}\right)=\min _{\bar{N}}\left(\bar{S}, \bar{U}_{1}\right)=\min _{\bar{N}}\left(\bar{U}, \bar{U}_{1}\right)=\overline{1} .
$$

Moreover, to obtain the second equality, we also use the fact that $\bar{U}_{1}$ covers a Sylow 2-subgroup of $\bar{P}$ and $|\bar{N}: \bar{P}|=2$. Now, by Lemma 2.3 , with $G=G, G_{1}=N, G_{2}=O_{2}(N)$ and $A=B=S$, by $O_{2}(N) \cap S^{x}=1$, we have $S \cap S^{y}=1$ for some $y \in G$. That is, $\min _{G}(S, S)=1$. A contradiction.

So, $S_{1} \neq U_{1}$. Therefore, and by $|\bar{N}: \bar{P}|=2$, the subgroup $S_{1}$ covers a Sylow 2 -subgroup of $\bar{N}$. Since $O_{2}(N) \leqslant \min _{G}(S, S)$, to describe the subgroup $\min _{G}(S, S)$ it is necessary to know its image $\overline{\min _{G}(S, S)}$ in $\bar{N}=N / O_{2}(N)$. We show that $\overline{\min _{G}(S, S)}=\min _{\bar{N}}(\bar{S}, \bar{S})$. 
Suppose that $D=S \cap S^{x} \in m$ does not lie in the preimage of $S_{2}$ in the $N$ of the subgroup $\min _{\bar{N}}(\bar{S}, \bar{S})$, which by virtue of Lemma 2.1 is isomorphic to the dihedral group of order 16 . Then $S_{2} \cap S_{1}=1$, since $S_{2}<S$, and $S_{1}<S^{x}$. From here $\min _{\bar{N}}(\bar{S}, \bar{S}) \cap \bar{S}_{1}=\overline{1}$. But this is impossible, because $\bar{S}_{1} \in \operatorname{Syl}_{2}(\bar{N})$. Thus, $D<S_{2}$ and, consequently, $\overline{\min _{G}(S, S)} \leqslant \min _{\bar{N}}(\bar{S}, \bar{S})$.

On the other hand, each element $\bar{D} \in \bar{m}$ is of order 2 by Lemma 2.1 and by definition $\bar{D}=\bar{S} \cap \bar{S}_{1}^{\bar{y}}$ for some $\bar{y} \in \bar{N}$. Therefore, for the preimage $D \leqslant S$ of the subgroup $\bar{D}$ we have $\left|D: O_{2}(N)\right|=2$. Hence, $D \leqslant S_{1}^{y} O_{2}(N)$ and $D \nless O_{2}(N)$, otherwise $\bar{S} \cap \bar{S}_{1}^{\bar{y}}=\overline{1}$. Therefore, $\left|D \cap S_{1}^{y}\right|=2$. Further, $D \cap S_{1}^{y}=D \cap\left(N \cap S^{x}\right)^{y}=D \cap S^{x y}$. We show that $D \cap S^{x y}=S \cap S^{x y}$. Indeed, from $\left|D \cap S_{1}^{y}\right|=2$, we obtain $D=\langle d\rangle O_{2}(N)$, where $d$ is an involution. Since $S^{x y} \cap O_{2}(N)=1$, then the image in $\bar{S}$ of the intersection $D_{1}=S \cap S^{x y}$ contains isomorphic to $D_{1}$ copy $\overline{D_{1}}$. Obviously, $D_{1}$ is also contained in the intersection $N \cap S^{x y}=\left(N \cap S^{x}\right)^{y}=S_{1}^{y}$, and subgroup $S_{1}^{y}$ is isomorphic to its image $\bar{S}_{1}^{\bar{y}} \in \operatorname{Syl}_{2}(\bar{N})$. Therefore, $D_{1} \simeq \bar{D}_{1} \leqslant \bar{D}$. Since $|\bar{D}|=2$, then $\left|\bar{D}_{1}\right|=2=\left|D_{1}\right|=\left|S \cap S^{x y}\right|$. Hence $D \cap S^{x y}=S \cap S^{x y} \in m$. So, we have the correspondence $\bar{D} \rightarrow\langle d\rangle=D \cap S_{1}^{y}=S \cap S^{x y} \in m$. Therefore the subgroup $\min _{G}(S, S)$ covers the subgroup $\min _{\bar{N}}(\bar{S}, \bar{S})$. Hence, $\min _{\bar{N}}(\bar{S}, \bar{S}) \leqslant \overline{\min _{G}(S, S)}$.

Thus, we have established that $\overline{\min _{G}(S, S)}=\min _{\bar{N}}(\bar{S}, \bar{S})$. Now Theorem 1 follows from Lemma 2.1.

Theorem 1 is proved.

\section{The proof of the Theorem 2}

So, by the hypothesis of the theorem, $G$ is a finite group, $\operatorname{Soc}(G) \simeq F_{4}(2), A, B$ are primary $p$-subgroup of $G$, and $S$ is a Sylow 2-subgroup of $G$.

$(1) \Rightarrow(2)$. Let $\operatorname{Min}_{G}(A, B) \neq 1$. Then also $\min _{G}(A, B) \neq 1$. In view of Theorem $\mathrm{B}(2)$ of [3], $G \simeq \operatorname{Aut}\left(F_{4}(2)\right)$ and the subgroups $A$ and $B$ are 2-groups. Without loss of generality we can

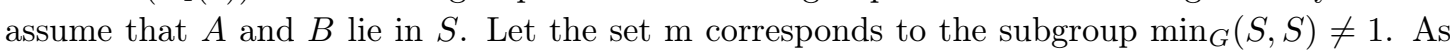
shown in the proof of Theorem 1, all elements of the set $m$ have order 2 . Therefore, if an element of the $m$ is not in $\min _{G}(A, B)$, then $\min _{G}(A, B)=1$, but this is impossible by assumption. Hence, $\min _{G}(S, S) \leqslant \min _{G}(A, B)$. Since $A$ and $B$ are 2-groups and $\left|S: \min _{G}(S, S)\right|=2$ by Theorem 1, then the subgroups $A$ and $B$ coincide with the subgroups $S$ or $\min _{G}(S, S)$.

We show that the pair $(A, B)=\left(\min _{G}(S, S), \min _{G}(S, S)\right)$ is excluded. Again, in view of Theorem 1

$$
\min _{G}(S, S)=O_{2}\left(P_{\{2,3\}}\right) \cdot \min _{L\langle\tau\rangle}\left(S_{1}, S_{1}\right) \leqslant O_{2}\left(P_{\{2,3\}}\right) \lambda L\langle\tau\rangle,
$$

where $S_{1}$ is a Sylow 2-subgroup of the group $L\langle\tau\rangle \simeq \operatorname{Aut}\left(A_{6}\right)$, and $\min _{L\langle\tau\rangle}\left(S_{1}, S_{1}\right) \simeq D_{16}$. By Lemma $3.2, S \cap S^{n_{0}}=\langle\tau\rangle$, therefore, $\min _{G}(S, S) \cap\left(\min _{G}(S, S)\right)^{n_{0}} \leqslant\langle\tau\rangle$. In particular, $O_{2}\left(P_{\{2,3\}}\right) \cap O_{2}\left(P_{\{2,3\}}\right)^{n_{0}}=1$. Since $n_{0} \tau=\tau n_{0}$, then $(L\langle\tau\rangle)^{n_{0}}=L\langle\tau\rangle$. Summarizing all of the above and applying Lemma 2.2, we obtain the existence of an element $g \in L\langle\tau\rangle$ such that $\min _{G}(S, S) \cap\left(\min _{G}(S, S)\right)^{g}=1$.

$(2) \Rightarrow(1)$. If $(A, B)=(S, S)$, then $\operatorname{Min}_{G}(A, B) \neq 1$ in view of Theorem 1.

Let $(A, B)=\left(\min _{G}(S, S), S\right)$. Suppose that $\operatorname{Min}_{G}(A, B)=1$. Then also $\min _{G}(A, B)=1$.

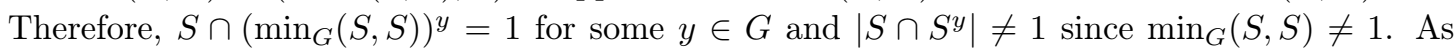
noted above, $\left|S: \min _{G}(S, S)\right|=2$ by Theorem 1 . Moreover, it follows from Theorem 1 that $S=\min _{G}(S, S) \lambda\langle i\rangle$ for any involution $i \in S \backslash \min _{G}(S, S)$. Therefore, $\left|S \cap S^{y}\right|=2$, otherwise $S \cap\left(\min _{G}(S, S)\right)^{y} \neq 1$. Thus, $S \cap S^{y} \in m$ and $S \cap S^{y} \leqslant \min _{G}\left(S^{y}, S^{y}\right)=\left(\min _{G}(S, S)\right)^{y}$. This is a contradiction. 
The case $(A, B)=\left(S, \min _{G}(S, S)\right)$ is considered similar to the case $(A, B)=\left(\min _{G}(S, S), S\right)$.

Theorem 2 is proved.

The first author was supported by the RNF (project 15-11-10025), Theorem 1, as well as agreements between the Russian Federation Ministry of Education and Science and Ural Federal University on 08/27/2013, number 02.A03.21.0006, Theorem 2. The work of the second author was supported by the RFBR (project 16-01-00707).

\section{References}

[1] V.I.Zenkov, On intersection of nilpotent subgroups in finite symmetric and alternating groups, Trudy IMM UrO RAN, 19(2013), no. 3, 144-149 (in Russian).

[2] V.I.Zenkov, Intersections of Abelian subgroups in finite groups, Matemat. zametki, 56(1994), no. 2, 150-152 (in Russian).

[3] V.I.Zenkov, Intersection of nilpotent subgroups in finite groups, Fundamental. i prikladnaya matematika, 2(1996), no. 1, 1-92 (in Russian).

[4] V.I.Zenkov, On intersection of nilpotent subgroups in finite groups with socle $L_{2}(q)$, Siberian Math. J., 57(2016), no. 6, 1280-1290 (in Russian).

[5] V.I.Zenkov, Ya.N.Nuzhin, On intersection of primary subgroups of odd order in finite almost simple groups, Fundamental. i prikladnaya matematika, 19(2014), no. 6, 115-123 (in Russian).

[6] R.Carter, Simple groups of Lie type, London, New York, Sydney, Toronto, Wiley and Sons, 1972.

[7] V.M.Levchuk, Automorphisms of unipotent subgroup of Chevalley groups, Algebra i Logika, 29(1990), no. 3, 315-338 (in Russian).

[8] N.Bourbaki, Groupes et algebres de Lie. VI-VI, Paris, Hermann, 1968.

\section{O пересечениях примарных подгрупп в группе $\operatorname{Aut}\left(\mathbf{F}_{4}(2)\right)$}

Виктор И. Зенков

Институт математики и механики УрО РАН Ковалевской, 16, Екатеринбург, 620990

Россия

Яков Н. Нужин

Институт математики и фундаментальной информатики Сибирский федеральный университет Свободный, 79, Красноярск, 660041

Россия

Показано, что в конечной группе $G$, изоморфной группе всех автоморфизмов группь Шевалле $F_{4}(2)$, существуют лищь три типа упорядоченных пар примарных подгрупп $A$ и $B$ с условием: $A \cap B^{g} \neq 1$ для любого $g \in G$. Приведено описание всех упорядоченных пар $(A, B)$ таких подгрупп с точностъю до сопряженности в группе $G$, в частности, доказано, что $A$ и $B$ являются 2-группами.

Ключевые слова: конечная группа, почти простая группа, примарная подгруппа. 